\title{
Green element method solutions to steady inverse contaminant transport problems
}

\author{
E. K. Onyari ${ }^{1} \&$ A. E. Taigbenu ${ }^{2}$ \\ ${ }^{1}$ Department of Civil Engineering, \\ University of South Africa, South Africa \\ ${ }^{2}$ School of Civil and Environmental Engineering, \\ University of the Witwatersrand, South Africa
}

\begin{abstract}
Inverse contaminant transport problems, unlike direct problems, may result in non-unique and unstable solutions because of the ill-conditioned nature of the coefficient matrix. In this work the Green element method (GEM) is used to solve steady inverse contaminant transport problems. The ill-conditioned, overdetermined system of equations that arises from the Green element discretization is solved by the least square method with the singular value decomposition technique and Tikhonov regularization. Two examples of steady inverse contaminant transport problems with constant and variable velocity are simulated by GEM with good prediction obtained for the concentration and fluxes.

Keywords: Green element method, steady inverse contaminant transport, convection-diffusion, singular value decomposition.
\end{abstract}

\section{Introduction}

Contaminant transport can be solved in a direct manner to determine concentration using forward models that are generally stable with well behaved solution spaces. These problems are said to be well-posed because their solutions satisfy the requirements of existence, uniqueness and stability. In contrast, solutions of inverse problems may exhibit non-uniqueness, non-existence and instability [1]. In recent years, significant developments in solving inverse illposed problems utilizing various solution techniques have been reported. Typical ill-posed problems include numerical differentiation of noisy data [2], the inverse 
heat conduction problem [3], interpretation of geophysical data [4], and the inverse problem of contaminant transport [5]. The inverse contaminant transport problem in groundwater is mainly involved with reconstructing the history of a contaminant. Various aspects that have been addressed include identifying the contaminant source location [6-10], estimating the number of possible pollution sources [10], estimating the release history [11-14], and recovering the historical spatial and temporal distribution of a contaminant [9]. Generally, inverse problems emerge when available measurements of concentration at some points and fluxes along a part of a boundary are used to predict medium parameters or concentration and/or fluxes or to locate pollution sources. In this paper, the Green element method $[15,16]$ is applied to steady inverse contaminant transport problems in which concentration and fluxes are determined at parts of the computational domain using available data.

\section{Inverse advection-dispersion contaminant transport}

The mathematical formulation of the inverse contaminant transport problem considered in this work is described by the differential equation

$$
\nabla \cdot(D \nabla C)-\nabla \cdot(\mathbf{V} C)=Q(x, y)
$$

where $\nabla=2 D$ gradient operator; $Q=$ source or sink; $C=$ contaminant concentration; $\mathbf{V}=\mathbf{i} u+\mathbf{j} v$ is the pore velocity vector; and $D=$ hydrodynamic dispersion. The specified boundary conditions are given as:

$$
\begin{gathered}
C(x, y)=f_{1} \text { on } \Gamma_{1} \\
-D \nabla C \cdot \mathbf{n}=q_{1} \text { on } \Gamma_{2} \\
C(x, y)=f_{3} \text { and }-D \nabla C \cdot \mathbf{n}=q_{3} \quad \text { on } \Gamma_{3}
\end{gathered}
$$

where $\mathbf{n}$ is the outward pointing normal on the boundary. The differential eqn. (1) applies to the domain $\Omega$ with the boundary $\Gamma=\Gamma_{1} \cup \Gamma_{2} \cup \Gamma_{3} \cup \Gamma_{4}$. The inverse problem requires the solution of the concentration and/or flux on the boundary $\Gamma_{4}$ from specified boundary conditions and pollutant concentration measurements provided at internal discrete points in the domain. For $N_{p}$ available measurements, a measured concentration value at an internal point $\left(x_{p} y_{p}\right)$ may be expressed as

$$
C\left(x_{p}, y_{p}\right)=C_{p}
$$

In order to solve the inverse problem presented in eqns. (1-5), it is expected that the number of generated discrete equations be equal to or greater than the number of unknowns. For this inverse problem to be solved by GEM eqn. (1) is rewritten as

$$
D \nabla^{2} C=-\nabla D \cdot \nabla C+\nabla \cdot(\mathbf{V} C)+Q
$$

Although GEM is amenable to heterogeneous media [15], only the homogenous case is addressed in this paper and hence eqn. (6) becomes

$$
\nabla^{2} C=\frac{1}{D}[\nabla \cdot(\mathbf{V} C)+Q]
$$


The partial differential eqn. (7) is transformed into an integral one by applying Green's second identity and utilizing the free space Green's function from the solution of $\nabla^{2} G=\delta\left(r-r_{i}\right)$ in the infinite space, that is $G=\ln \left(r-r_{i}\right)$. This integral equation is

$$
-\lambda C_{\left(r_{i}\right)}+\iint_{\Omega} G\left[\frac{1}{D}(\nabla \cdot(\mathbf{V} C)+Q)\right] d A+\int_{\Gamma}\left(C \frac{\partial G}{\partial n}-G \frac{\partial C}{\partial n}\right) d s=0
$$

The discretized form of eqn. (8) is achieved by discretizing the computational into polygonal elements (rectangular elements are used in this work) and interpolating the quantities $C, \mathbf{V}$ and $Q$ by basis functions of the Lagrange family, that is $C \approx N_{j} C_{j}$ ( $N_{j}$ are linear interpolating functions). The discrete element equation for each element $\Omega^{e}$ and boundary $\Gamma^{e}$ is

$$
\left.R_{i j} C_{j}+L_{i j} q_{j}+U_{i j k} u_{j} C_{k}+Y_{i j k} v_{j} C_{j}\right)+f_{i}=0
$$

in which $q=\partial C / \partial n$, the vector $f_{i}$ depends on whether the sources/sinks are point or distributed, and the elemental matrices are given as

$$
\begin{aligned}
& R_{i j}=\int_{\Gamma^{e}} N_{j} \nabla G_{i} \cdot \mathbf{n} d s-\delta_{i j} \lambda, \quad L_{i j}=\int_{\Gamma^{e}} N_{j} G_{i} d s, \\
& U_{i j k}=\iint_{\Omega^{e}} G_{i} N_{j} \frac{\partial N_{k}}{\partial x} d A, \quad Y_{i j k}=\iint_{\Omega^{e}} G_{i} N_{j} \frac{\partial N_{k}}{\partial y} d A
\end{aligned}
$$

The elemental integrations in eqn. (10) are done analytically. Aggregating the discrete element eqn. (9) for all elements used to discretize the computational domain gives a matrix equation

$$
E_{i j} C_{j}+B_{i j} q_{j}+F_{i}=0
$$

Incorporating the boundary conditions in eqns. (2)-(4) into eqn. (1) yields the global matrix equation

$$
\mathbf{A w}=\mathbf{b}
$$

where $\mathbf{A}$ is an $M \times N$ matrix, with $M$ being the number of nodes in the computational domain which is the same as the number of discrete equations generated. $N$ are the nodal unknowns, which correspond to the primary variable or its flux at the external nodes, and the primary variable in the internal nodes. $\mathbf{w}$ is an $N \times 1$ vector of nodal unknowns; $\boldsymbol{b}$ is an $M \times 1$ vector of known quantities. For inverse problems eqn. (12) is an over-determined, ill-conditioned system of equations. As an over-determined, a least square solution is sought, while A is decomposed by the singular value decomposition (SVD) technique according to [17]

$$
\mathbf{A}=\mathbf{U D V}^{T}=\sum_{i=1}^{N} \sigma_{i} u_{i} v_{i}^{T}
$$

where $\mathbf{U}$ is an $M \times M$ orthogonal matrix, $\mathbf{V}$ is an $N \times N$ orthogonal matrix, and $\boldsymbol{D}$ is an $M \times N$ diagonal matrix with $N$ non-negative diagonal elements $\left(\mathbf{D}=\operatorname{diag}\left(\sigma_{1}\right.\right.$, $\left.\sigma_{2} \ldots \sigma_{N}\right)$ ) which satisfy the condition: $\sigma_{1}>\sigma_{2}>\cdots>\sigma_{N}>0$. The least square solution of eqn. (12) requires the minimization of the Euclidean norm $\|\mathbf{A w}-\mathbf{b}\|^{2}$ which gives 


$$
\mathbf{w}=\left(\mathbf{A}^{T} \mathbf{A}\right)^{-1}\left(\mathbf{A}^{T} \mathbf{b}\right)=\sum_{i=1}^{N} \frac{u_{i}^{T} \mathbf{b}}{\sigma_{i}} v_{i}
$$

The small singular values cause instability in the solution of $\mathbf{w}$, and this can be ameliorated by using, for instance, the zero order Tikhonov regularization technique which requires the minimization of the Euclidean norm $\| \mathbf{A w}-$ $\mathbf{b}\left\|^{2}+\alpha^{2}\right\| \mathbf{w} \|^{2}$ that yields the solution for $\mathbf{w}$

$$
\mathbf{w}(\alpha)=\sum_{i=1}^{N} \frac{\sigma_{i}}{\alpha^{2}+\sigma_{i}^{2}} u_{i}^{T} \mathbf{b} v_{i}
$$

where $\alpha$ is the regularization parameter, and the factor $\sigma_{i} /\left(\alpha^{2}+\sigma_{i}^{2}\right)$ dampens the contribution of the small singular values. The choice of $\alpha$ is critical as too small values may retain the instability in the numerical solution or too large values will result in smooth solutions that do not reflect the physics of the problem.

\section{Numerical examples solved using GEM and SVD}

Two numerical examples of convection-diffusion contaminant transport are simulated. The first example has constant velocity, while the second example has variable velocity.

\subsection{Example 1: 1-D inverse steady convection-diffusion problem with constant velocity}

This problem has been solved as a direct problem [18]. It is a simple 1-D convection-diffusion problem with prescribed boundary conditions $C(x=0)=1$ and $C(x=1)=2$. The velocity is in the $x$-direction and it is uniform, and the dispersion coefficient is taken as unity, thus the analytical solution is given as

$$
C=2-\frac{1-e^{u(x-1)}}{1-e^{-u}}
$$

In this work the problem is posed as an inverse problem and analyzed in a 2$\mathrm{D}$ rectangular domain with no flux imposed on the top and bottom boundaries, the concentration and flux are specified along $x=1$ but no data along the boundary $x=0$ which is an $\Gamma_{4}$ boundary. Two cases are considered to assess the influence of available measured concentration data in the computational domain on the solution. In case (i) the concentration is given at $x=0.2$ and $x=0.8$, and in case (ii) at $x=0.6$ only. The computational domain is discretized into 5,10 and 20 uniform elements. The concentration profiles for case (i) and case (ii) are respectively presented in figs. 1(a) and 1(b) for the three discretizations used in the GEM simulations. The results indicate good prediction by GEM of the concentration at the $\Gamma_{4}$ boundary $x=0$. However the GEM with 5 elements is unable to correctly capture the concentration profile close to the boundary $x=1$ because of the inability of the coarse discretization to correctly capture the higher gradient of the concentration profile. The flux $q(x=0)$ is well estimated in all the cases as shown in table 1 . The relative error for the calculated flux $q(x=0)$ is presented in fig. 2, decreases with increase in the number of elements. 
Furthermore, the relative error is smaller for case (i) than case (ii), and this is due to the higher number of available data and their location.

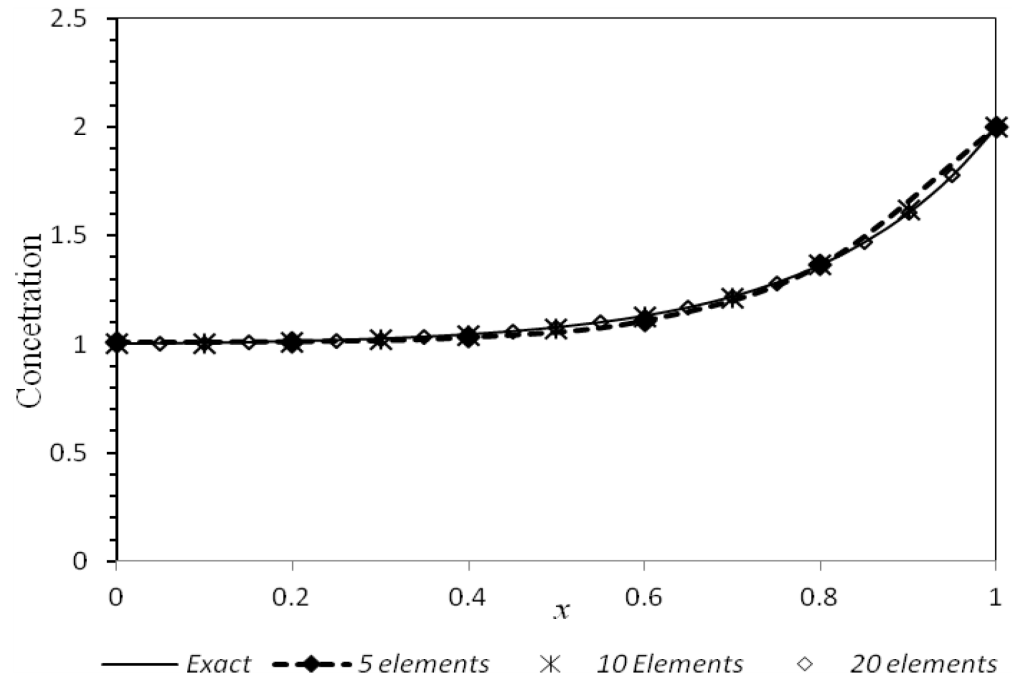

(a)

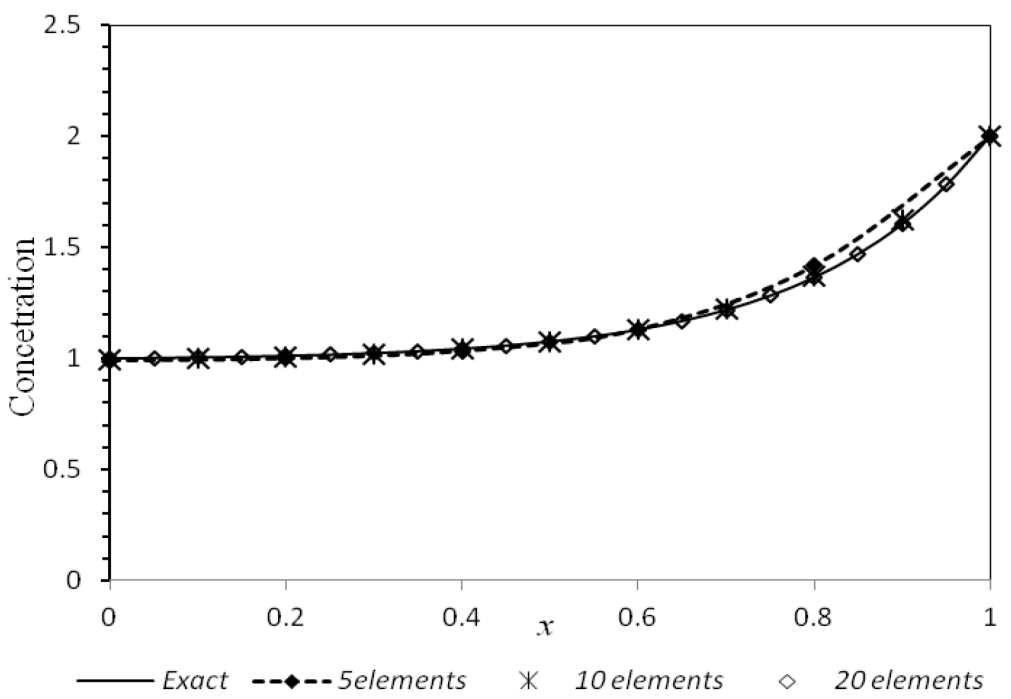

(b)

Figure 1: Inverse GEM and exact solutions for $C(x)$ with 5, 10 and 20 elements for Example 1: (a) case (i), (b) case (ii). 
98 Boundary Elements and Other Mesh Reduction Methods XXXV

Table 1: Computed flux at $x=0$ for Example 1 different element discretizations.

\begin{tabular}{|c|l|l|l|c|c|}
\hline \multirow{2}{*}{ Exact } & \multicolumn{5}{|c|}{ Inverse GEM solutions } \\
\cline { 2 - 6 } & \multirow{2}{*}{$\begin{array}{l}\text { No. of } \\
\text { elements }\end{array}$} & \multicolumn{2}{|c|}{ Case (i) } & \multicolumn{2}{c|}{ Case (ii) } \\
\cline { 3 - 6 } & & $q(x=0)$ & $\alpha$ & $q(x=0)$ & $\alpha$ \\
\hline 0.033918 & 5 & 0.033645 & $5.3 \times 10^{-3}$ & 0.033252 & $1.1 \times 10^{-2}$ \\
\hline 0.033918 & 10 & 0.031405 & $10^{-4}$ & 0.033633 & $2.5 \times 10^{-3}$ \\
\hline 0.033918 & 20 & 0.033227 & $10^{-4}$ & 0.033598 & $10^{-4}$ \\
\hline
\end{tabular}

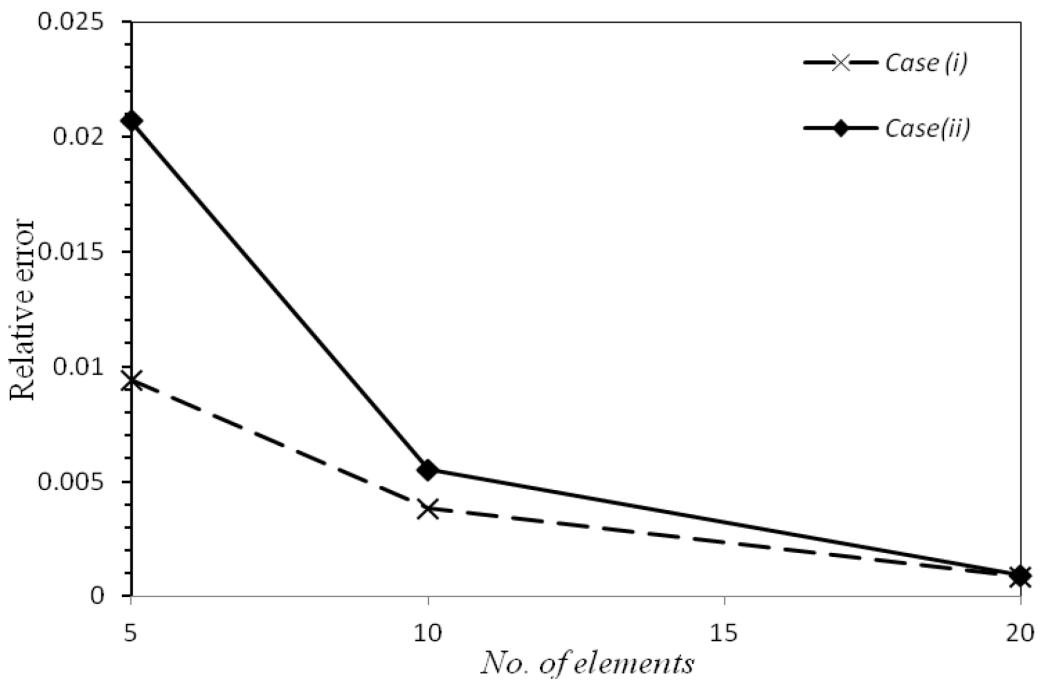

Figure 2: $\quad$ Relative error of inverse GEM solution for $q(x=0)$ of Example 1 for 5,10 and 20 elements.

\subsection{Example 2: 1-D steady convection-diffusion transport with variable velocity}

This is an example governed by eqn. (1) with contaminant transport in the $x$ direction and with variable velocity. Its direct formulation specifies the boundary conditions: $C(x=0)=C_{0}$, and $C(x=1)=C_{1}$. The velocity field in the $x$ direction is given as

$$
u=\ln \frac{C_{1}}{C_{0}}+k\left(x-\frac{1}{2}\right)
$$


The values of $k$ are taken as 10 and 40 , while $C_{0}=300$ and $C_{1}=10$. Taking the hydrodynamic dispersion coefficient as unity, the analytical solution is given as

$$
C=C_{0} \exp \left\{\frac{k}{2} x^{2}+\left(\ln \frac{C_{1}}{C_{0}}-\frac{k}{2}\right) x\right\}
$$

In this work, this example is simulated in 2-D as an inverse problem in which the boundary along $x=0$ is a $\Gamma_{4}$ boundary where the $C$ and $q$ are unknown, while the boundary along $x=1$ is a $\Gamma_{3}$ boundary where $C$ and $q$ are specified, and concentration data are provided at $x=0.1,0.3,0.5,0.7$ and 0.9 . The upper and lower boundaries of the computational domain are no flux boundaries. The computational domain is discretized uniformly into 10,20 and 40 rectangular elements. A non-uniform discretization of 14 elements is also employed in order to capture the steep gradient of the concentration profile close to $x=0$. The lengths of these rectangular elements in the $x$-direction are: $0.025,0.025,0.025$, $0.025,0.05,0.05,0.1,0.1,0.1,0.1,0.1,0.1,0.1$ and 0.1 . The concentration profiles for $k=10$ and $k=40$ from GEM simulations are presented with the exact solution in figs. 3(a) and 3(b), and they show that GEM gives better prediction of $C(x=0)$ for the less convective case $(k=10)$ than the more convective one $(k=40)$. For both cases, the accuracy of the GEM solutions is enhanced with increase in the number of uniform elements incorporated in the simulations. However, using 14 non-uniform elements produces solutions with accuracy comparable to those with 20 and 40 uniform elements. The GEM solutions for the flux $q(x=0)$ are presented in table 2 for the two convective cases, and their errors relative to the exact solution are presented in fig. 4. As earlier observed with the GEM solution of $C(x=0)$, that for the flux $q(x=0)$ is similar in that the solution with 14 non-uniform elements is comparable to that with 40 uniform elements.

\section{Conclusions}

This paper has presented Green element solutions of the inverse convectiondiffusion contaminant transport equation. The GEM discretization of the differential equation gives rise to an overdetermined, ill-conditioned global matrix that is solved by the least square method with Tikhonov regularization and the SVD for the decomposition of the matrix. Using two numerical examples of steady 1-D transport problems that were simulated by GEM in 2-D, the influences of the regularization parameter value, the level of discretization, the distribution of measured data, and the strength of convection on the numerical accuracy were assessed. The level of accuracy of the numerical solutions achieved for these examples demonstrate that GEM is capable of simulating inverse steady contaminant transport problems. 


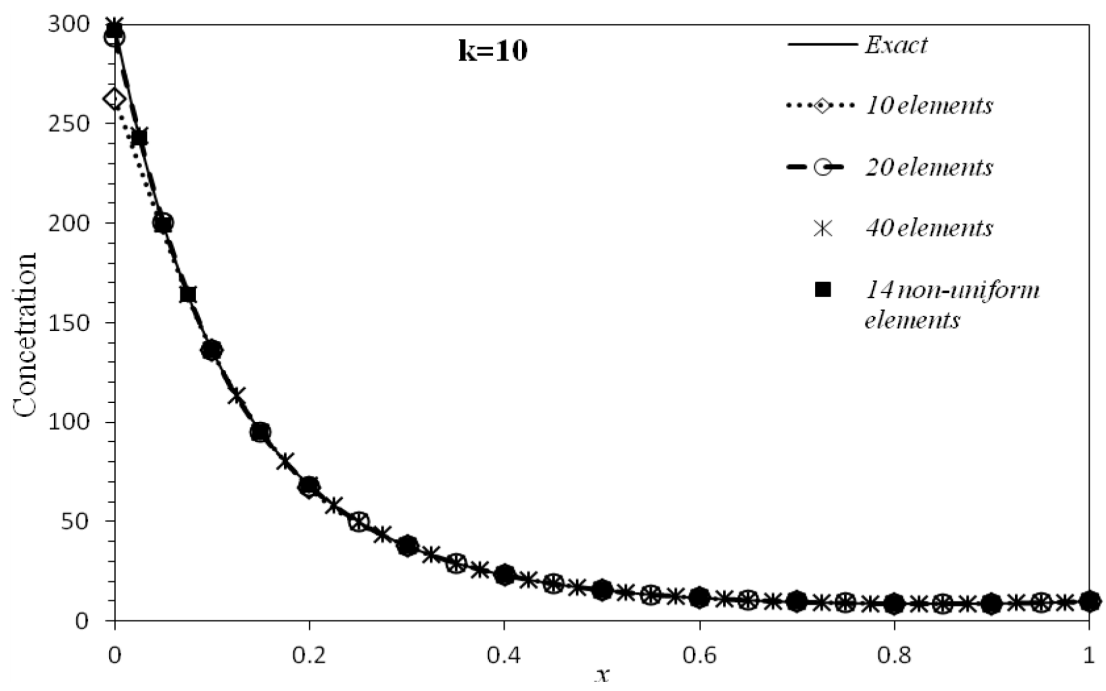

(a)

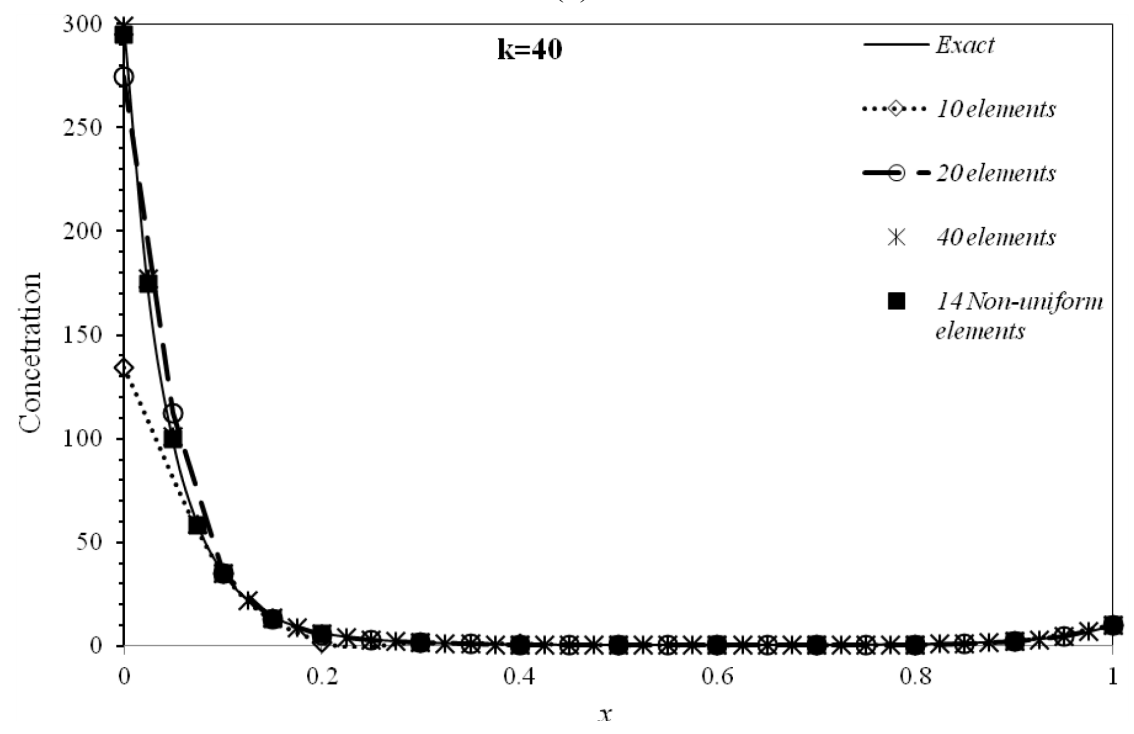

(b)

Figure 3: Inverse GEM and exact solutions for $C(x)$ with 5, 10, 20 uniform elements, and 14 non-uniform elements for Example 2: (a) $k=10$, (b) $k=40$. 
Table 2: $\quad$ Computed flux at $x=0$ for $k=10$ and $k=40$.

\begin{tabular}{|c|c|c|c|c|c|c|}
\hline \multicolumn{4}{|c|}{ Convective value $k=10$} & \multicolumn{3}{|c|}{ Convective value $k=40$} \\
\hline \multirow[t]{2}{*}{$\begin{array}{c}\text { Exact } \\
q(x=0)\end{array}$} & \multicolumn{3}{|c|}{ Inverse GEM solutions } & \multirow[t]{2}{*}{$\begin{array}{c}\text { Exact } \\
q(x=0)\end{array}$} & \multicolumn{2}{|c|}{$\begin{array}{c}\text { Inverse GEM } \\
\text { solutions }\end{array}$} \\
\hline & $\begin{array}{l}\text { No. of } \\
\text { elements }\end{array}$ & $q(x=0)$ & $\alpha$ & & $q(x=0)$ & $\alpha$ \\
\hline-2520.36 & 10 & -2179.61 & $10^{-4}$ & -7020.36 & -3120.01 & $10^{-5}$ \\
\hline-2520.36 & 20 & -2461.45 & $10^{-5}$ & -7020.36 & -6414.73 & $10^{-6}$ \\
\hline-2520.36 & 40 & -2512.73 & $10^{-5}$ & -7020.36 & -6998.45 & $10^{-6}$ \\
\hline-2520.36 & $\begin{array}{l}14 \text { (non- } \\
\text { uniform) }\end{array}$ & -2478.95 & $10^{-5}$ & -7020.36 & -6889.44 & $10^{-6}$ \\
\hline
\end{tabular}

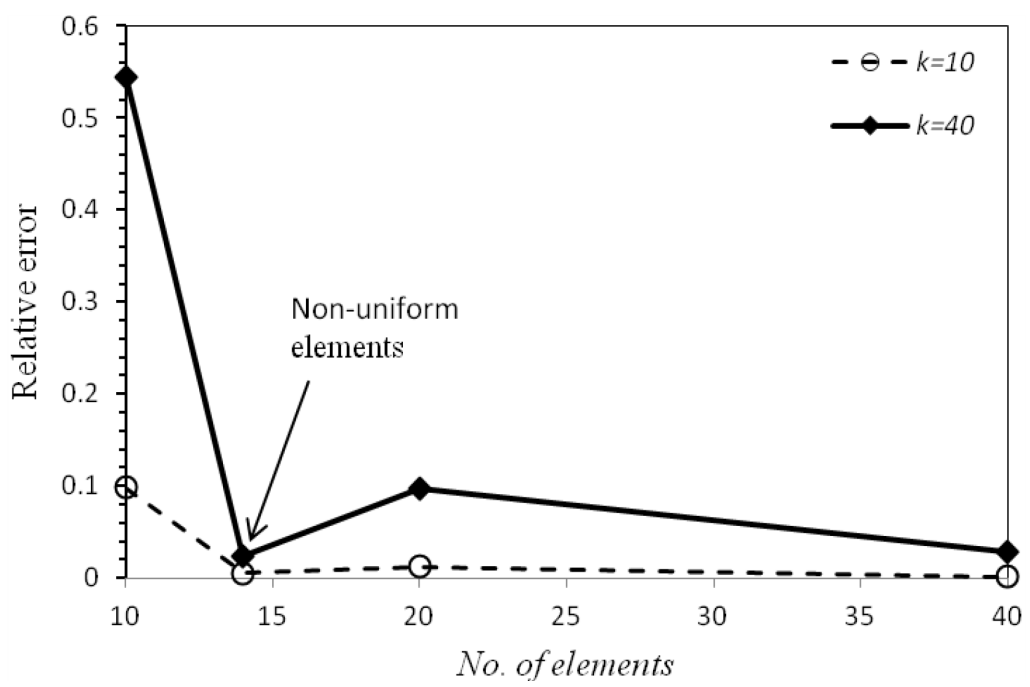

Figure 4: Relative error of inverse GEM solution for Example 2 with 5, 10, 20 uniform elements and 14 non-uniform elements.

\section{References}

[1] Sun, N.-Z., Mathematical modelling of Groundwater pollution. SpringerVerlag: New York, USA, 1996.

[2] Andersses, R.S. and Bloomfield, P. Numerical differentiation procedures for non exact data. Numerical mathematics, 22, pp. 157-182, 1974.

[3] Beck, J., Blackwell, B. and Clair, C.R., Inverse Heat Conduction, Illposed problems. Wiley Interscience: New York, 1985.

[4] Backus, G. and Gilbert, F., Uniqueness in the inversion of inaccurate gross Earth data. Philos. Trans. R. Soc. London, ser A, 266, pp. 123-192, 1970. 
[5] Yeh, W. W.-G. Review of parameter identification procedures in groundwater hydrology: The inverse problem. Water Resources Research, 22(2), pp. 95-108, 1986.

[6] Gorelick, S.M., Evans, B.and Remson, I. Identifying sources of groundwater pollution: An optimisation approach. Water Resources Research, 19(3), pp. 779-790, 1983.

[7] Wagner, B.J., Simultaneous parameter estimation and contaminant source characterisation for coupled groundwater flow and contaminant flow and contaminant transport modelling. Journal of Hydrology, 135, pp. 275-303, 1992.

[8] Mahar, P.S. and Datta, B. Identification of pollution sources in transient groundwater system. Water Resources Research, 14(6), pp. 209-227, 2000.

[9] Michalak, A.M. and Kitanidis, P.K., Estimation of historical groundwater contaminant distribution using adjoint state method applied to geostatistical inverse modelling. Water Resources Research, 40, 2004.

[10] Ayvaz, M.T., A linked simulation-optimization model for solving the unknown groundwater pollution source identification problems. Journal of Contaminant Hydrology, 117, pp. 46-59, 2010.

[11] Skaggs, T.H. and Kabala, Z.J. Recovering the release history of a groundwater contaminant. Water Resources Research, 30(1), pp. 71-79, 1994.

[12] Skaggs T.H. and Kabala, Z.J. Recovering the history of a groundwater contaminant plume: Method of Quasi-Reversibility. Water Resources Research, 31(11), pp. 2669-2673, 1995.

[13] Alapati, S., and Kabala, Z.J., Recovering the release history of a groundwater contaminant using a non-linear least squares method. Hydrological processes, 14, pp. 1003-1016, 2000.

[14] Tian, N., Jun Sun, Wenbo, X. and Choi-Hong, L, An improved quantum behaved particle swarm optimization with perturbation operator and its application in estimating groundwater contaminant source. Inverse Problems in Science and Engineering, 19(2), pp. 181-202, 2011.

[15] Taigbenu, A.E., The Green Element Method. Kluwer Academic Publishers: USA, 1999.

[16] Taigbenu, A.E., Enhancement of the accuracy of the Green element method: Application to potential problems. Engineering Analysis with Boundary Elements 36, pp. 125-136, 2012.

[17] Heath, M.T., Scientific computing: An introductory survey. McGraw-Hill. USA, 1997.

[18] Qui, Z.H., Wrobel, C, and Power, H., Numerical solution of convective diffusion problems at high Peclet number using Boundary elements. International Journal for Numerical Methods in Engineering. 41, pp. 899914, 1998. 